\title{
Development of Short-term Traffic Volume Prediction Models for Adaptive Traffic Control
}

\author{
Yiming Bie ${ }^{1, a}$, Menglin Yang ${ }^{2, b}$, Yulong $\mathrm{Pei}^{1, \mathrm{c}}$ \\ ${ }^{1}$ School of Transportation Science and Engineering, Harbin Institute of Technology, \\ Harbin, 150090, China \\ ${ }^{2}$ School of Transportation, Southeast University, Nanjing, 210018, China \\ aemail:yimingbie@126.com, bemail:lily1919lily@163.com, cemail:346319930@qq.com
}

\begin{abstract}
Keywords: Short-term Traffic Volume, Prediction Method, Statistics interval, Adaptive Traffic Control
\end{abstract}

\begin{abstract}
This paper aims at establishing an accurate short-term traffic volume prediction method by introducing calculations and characteristics of triple moving average method, single exponential smoothing method, and double exponential smoothing method. Based on field data collected from intersections in Fuzhou, the prediction accuracy of three prediction methods above was calculated respectively at 5-minute, 10 -minute and 15-minute statistics intervals and analyzed. Analysis results indicate that prediction methods and statistics intervals have significant impacts on the prediction accuracy. To be specific, the prediction accuracy of double exponential smoothing method is higher than other two methods; The accuracy at 10-minute statistics intervals is higher than that at other two intervals. The research results will provide an accurate volume prediction method for adaptive traffic control.
\end{abstract}

\section{Introduction}

Adaptive traffic control is an effective method to improve operation efficiency of traffic flow and alleviate traffic congestion. It generally obtains traffic volume through real-time detection of passing vehicles, by loop detectors laid on each approach lane of intersections. Automatic detection of traffic volume is pivotal for applying adaptive traffic signal control [1][2]. Traffic control systems normally record traffic volume passing over loop detectors and optimize signal timing plans at fixed time intervals (e.g. 5 minute). To get optimal timing plans for the next interval, systems need to predict volumes based on volumes of several previous intervals. This is short-term traffic volume prediction problem of adaptive traffic control. With higher prediction accuracy, optimized signal timing plans will be able to adapt better to traffic flow state of the next interval [3].

Numerous scholars have done researches on short-term traffic volume prediction and achieved abundant results [4][5]. Vlahogianni et al. [6] extended past research by providing an advanced, genetic algorithm based, multilayered structural optimization strategy that could assist both in the proper representation of traffic flow data with temporal and spatial characteristics as well as in the selection of the appropriate neural network structure. Further, they evaluated the performance of the developed network by applying it to both univariate and multivariate traffic flow data from an urban signalized arterial. Park et al. [7] applied a radial basis function (RBF) neural network to time-series forecasting. The test results of an RBF neural network in forecasting short-term freeway traffic volumes were provided. Real observations of freeway traffic volumes from the San Antonio TransGuide System had been used in their experiments. For comparison of forecasting performances, Taylor series, exponential smoothing method (ESM), double exponential smoothing method, and backpropagation neural network were also designed and tested.

Although plentiful studies have been conducted, prediction methods are still complicated and difficult for engineering application, which needs concise, fast and accurate methods to improve real-time performance of systems. This paper made predictions with triple moving average method, single exponential smoothing method, and double exponential smoothing method, compared their performance and selected the optimal prediction method. 


\section{Prediction Models}

Loop detectors are laid in each approach lane of signalized intersections. Arrival volume of each approach lane is affected by route choices of drivers, departure time and road traffic management plans which are highly random. It is difficult to establish models based on these elements. However, controllers collect arrival volume passing over loop detectors of a certain lane at 5-minute intervals and traffic flow states of adjacent intervals share similarities. Thus, traffic flow of the next interval can be predicted based on traffic volumes of several previous intervals, which is converted to traffic volume time-series prediction. The effect of traffic flow random fluctuation gets more significant with shorter prediction time interval, which would contribute to larger prediction errors. The traffic volume of the interval next to current moment is predicted respectively at 5-minute, 10-minute, and 15-minute statistics intervals in this paper and prediction errors are analyzed to select the interval with the least prediction error.

Triple moving average method. The formula of triple moving average method can be expressed as Eq.(1), which indicates that average volume of arriving vehicles in the past three intervals can be used as predicted volume of arriving vehicles in the next interval.

$$
\hat{X}_{t+1}=\left(X_{t}+X_{t-1}+X_{t-2}\right) / 3
$$

where: $\hat{X}_{t+1}$ - predicted volume of arriving vehicles at certain approach lane in interval $t+1$;

$X_{t}$ — the volume of actually arriving vehicles at certain approach lane in interval $t$.

Single exponential smoothing method. The formula of single exponential smoothing method can be expressed as Eq.(2),

$$
\hat{X}_{t+1}^{(1)}=\alpha X_{t}+\alpha(1-\alpha) X_{t-1}+\alpha(1-\alpha)^{2} X_{t-2}+\ldots
$$

where: $\hat{X}_{t+1}^{(1)}$ - predicted volume of arriving vehicles at certain approach lane in interval $t+1$. Superscript (1) stands for single exponential smoothing; $\alpha$ - smoothing parameter, $1<\alpha<1$.

volume of actually arriving vehicles in several previous intervals are considered and intervals nearer to current moment are assigned to higher weight in single exponential smoothing method. $\alpha$ is advised to be taken as 0.7 . Then weights for volume of actually arriving vehicles in interval $t, t-1$, $t-2$, and $t-3$ are $0.7,0.21,0.063$, and 0.0189 , of which the accumulated sum is 0.9919 and approximately 1.0. Thus, single exponential smoothing method only utilizes volume of actually arriving vehicles in the four previous intervals when $\alpha=0.7$ :

$$
\hat{X}_{t+1}^{(1)}=0.7 X_{t}+0.21 X_{t-1}+0.063 X_{t-2}+0.0189 X_{t-3}
$$

Double exponential smoothing method. The formula of double exponential smoothing method can be expressed as Eq.(4), which indicates that predicted value in interval $t+1$ is the weighted sum of predicted value after single exponential smoothing in interval $t+1$ and predicted value after double exponential smoothing in interval $t$.

$$
\hat{X}_{t+1}^{(2)}=\alpha \hat{X}_{t+1}^{(1)}+(1-\alpha) \hat{X}_{t}^{(2)}
$$

where: $\hat{X}_{t+1}^{(2)}$ - predicted volume of arriving vehicles at certain approach lane in interval $t+1$. Superscript (2) stands for double exponential smoothing.

$\alpha$ is advised to be taken as 0.7 as well. When $t=1$, Eq.(4) can be expressed as:

$\hat{X}_{2}^{(2)}=0.7 \hat{X}_{2}^{(1)}+0.3 \hat{X}_{1}^{(2)}$

Here $\hat{X}_{1}^{(2)}$ can be replaced by the actual arrival volume in interval 1 .

\section{Prediction result analysis and evaluation}

Traffic flow data, collected from the intersection of Wuyi Road and JinTai Road in Fuzhou during 6:00 to 24:00 on a certain day, is utilized to test three prediction models above. Loop detectors are laid 40 meters behind stop lines in each approach lane of the intersection. Volumes of passing vehicles are recorded every 5 minutes.

Prediction results. Four approach lanes of this intersection are selected to make predictions, which are Lane 1 of north leg, Lane 2 of north leg, Lane 3 of south leg and Left lane of west leg. 
Generally speaking, random fluctuation of traffic flow is more significant during flat and low peak period which contributes to larger prediction errors, while the arrival of traffic flow is steady in rush hours and prediction errors are smaller. In addition, during flat and low peak period, larger prediction errors of traffic flow has less impact on trips of travelers because of a big surplus in capacity of road network, slight delay and less queuing vehicles in intersections. On the contrary, traffic congestion normally occurs in road network during rush hours and drivers are sensitive to travel information, during which period traffic flow prediction needs high accuracy.

Considering the difference in prediction errors and the sensitivities between flat and low peak period and rush hours, two categories of errors are recorded: prediction errors of all sets of data collected from 6:00 to 24:00 and errors of data from morning rush hour 7:30-8:30 and evening rush hour 18:00-19:00. Besides, average, maximum and minimum errors are calculated of each category.

Concrete prediction results are demonstrated as follows.

1) Triple moving average method

Take Lane 3 of south leg as an example. The total average prediction error based on triple moving average method is $9.85 \%$ at 10 -minute statistics intervals and the average prediction error of 2 peak hours is $14.22 \%$, which are both smaller than prediction errors at 5 -minute and 15 -minute intervals. For Lane 1 of north approach and left lane of west approach, the average prediction errors of whole time and 2 peak hours are also minimum at 10 -minute intervals.

2) Single exponential smoothing method

Take Lane 1 of north leg as an example. By applying single exponential smoothing method, total average prediction errors at 5-minute, 10 -minute and 15-minute intervals are respectively $17.43 \%$, $11.67 \%$ and $12.01 \%$, which indicates that the prediction error is minimum at 10 -minute intervals. Furthermore, the average prediction error of 2 peak hours is not larger than the total average prediction error because traffic volume is high and quite steady during rush hours. It is beneficial for the application of time- series prediction methods.

3) Double exponential smoothing method

Take Lane 1 of north leg as an example. The maximum of the total average prediction error based on double exponential smoothing method is $266.56 \%$ at 15 -minute intervals, which is far from the acceptable prediction error for engineering. The main reason is the relatively wide random fluctuation of traffic flow during flat and low peak period. For 2 peak hours, maximum prediction errors at 5-minute, 10 -minute and 15 -minute intervals are respectively $64.81 \%$, $26.30 \%$ and $87.33 \%$. According to the analysis above, statistics intervals have an significant impact on prediction errors.

Results analysis. Through analyzing prediction results above, we can conclude the following rules:

1) Selection of prediction models has an significant impact on prediction accuracy.

Triple moving average method, single exponential smoothing method and double exponential smoothing method are compared with average error as evaluation indicator. It is found that traffic volume prediction errors are relatively small when double exponential smoothing method is adopted. Besides, the prediction accuracy of single exponential smoothing method is higher than that of triple moving average method.

2) Selection of statistics interval has an significant impact on prediction accuracy.

Traffic volume prediction errors at 5-minute, 10-minute and 15-minute statistics intervals are calculated and the results indicate that statistics intervals affect prediction accuracy as well. Under nearly almost conditions, the prediction accuracy at 10-minute intervals is the highest, which means that at the present moment, the arrival volume of approach lanes predicted for next 10 minutes based on historical data are the most accurate. This may be because 5-minute interval is too short to eliminate the random fluctuation of traffic flow while 15-minute interval is too long that time delay is prolonged in prediction which causes large prediction errors.

3) Traffic flow in city has wide random fluctuation which causes the difficulty to obtain pretty high prediction accuracy.

There exists large difference among arrival traffic volume in adjacent statistics intervals of the same approach lane which demonstrates that traffic flow has wide random fluctuation. As illustrated 
from Table 1 to Table 3, when prediction errors are smaller than 15\%, the prediction accuracy might be deemed high. Thus. It is tough to establish prediction methods with quite high accuracy for adaptive traffic control system.

\section{Conclusion}

Short-term traffic volume prediction of approach lanes of intersections is the basis of adaptive traffic control. Based on the field data collected in Fuzhou, triple moving average method, single exponential smoothing method and double exponential smoothing method are adopted to calculate short-term traffic volume prediction errors respectively at 5-minute, 10-minute and 15-minute statistics intervals. Analysis indicates that prediction methods and statistics intervals have an significant impact on prediction results. To be specific, higher prediction accuracy can be obtained by utilizing double exponential smoothing method at 10-minute intervals.

\section{Acknowledgement}

This work was supported by the National Natural Science Foundation of China under Grant NO. 61304198; the China Postdoctoral Science special Foundation under Grant NO. 2014T70351 and the China Postdoctoral Science Foundation funded projects under Grant NO. 2013M530159.

\section{References}

[1] Wang L, Bie Y. An adaptive model for calculating the correlation degree of multiple adjacent signalized intersections [J]. Mathematical Problems in Engineering, vol. 2013, Article ID 247184, 13 pages, 2013.

[2] Bie Y, Wang D, Qu X. Modelling correlation degree between two adjacent signalised intersections for dynamic subarea partition [J]. IET Intelligent Transport Systems, 2013, 7(1): 28-35.

[3] Bie Y, Wang D, Qi H. Prediction model of bus arrival time at signalized intersection using GPS data [J]. Journal of Transportation Engineering, 2011, 138(1): 12-20.

[4] Bie Y, Liu Z, Ma D, et al. Calibration of platoon dispersion parameter considering the impact of the number of lanes [J]. Journal of Transportation Engineering, 2013, 139(2): 200-207.

[5] Vlahogianni E I, Golias J C, Karlaftis M G. Short - term traffic forecasting: Overview of objectives and methods [J]. Transport reviews, 2004, 24(5): 533-557.

[6] Vlahogianni E I, Karlaftis M G, Golias J C. Optimized and meta-optimized neural networks for short-term traffic flow prediction: A genetic approach [J]. Transportation Research Part C: Emerging Technologies, 2005, 13(3): 211-234.

[7] Park B, Messer C J, Urbanik II T. Short-term freeway traffic volume forecasting using radial basis function neural network [J]. Transportation Research Record: Journal of the Transportation Research Board, 1998, 1651: 39-47. 